\title{
E agora Dr.? O pediatra diante do diagnóstico do Transtorno do Espectro Autista
}

¿Y ahora doctor? El pediatra delante del diagnóstico del Transtorno del Espectro Autista

And now doctor? Pediatra faces the diagnosis of Autistic Spectrum Disorder

Maria Evanir Vicente Ferreira

Centro Universitário Franciscano - Rio Grande do Sul - Brasil

Luciane Najar Smeha

Centro Universitário Franciscano - Rio Grande do Sul - Brasil

\section{RESUMO}

O diagnóstico do Transtorno do Espectro Autista é realizado pela avaliação clínica, levando em consideração os critérios estabelecidos pelo Manual Diagnóstico e Estatístico de Transtornos Mentais (DSM V). O objetivo deste estudo foi identificar as percepções dos médicos pediatras sobre o diagnóstico e intervenção precoce nesse transtorno. Participaram do estudo oito pediatras. Os dados foram coletados com a utilização de um questionário sóciodemográfico e uma entrevista semiestruturada. Para analisar os dados foi utilizada a técnica de análise de conteúdo. Os resultados mostraram que apesar dos avanços no interesse e no conhecimento do diagnóstico do TEA pelos pediatras, ainda não foi suficiente para que eles realizem o referido diagnóstico e oportunizem a intervenção precoce.

Palavras-chave: Transtorno do espectro autista. Diagnóstico. Intervenção precoce.

\section{RESUMEN}

El diagnóstico de Autism Spectrum Disorder se realiza mediante una evaluación clínica, teniendo en cuenta los criterios establecidos por el Diagnostic and Statistical Manual of Mental Disorders (DSMV). El objetivo de este estudio fue identificar las percepciones de los pediatras sobre el diagnóstico y la intervención temprana en este desorden. Eight pediatría en el estudio. Los datos se utilizaron utilizando la sociodemográfica cuestionnaire y la entrevista semi-escrita. Para analizar la fecha, se utilizó la técnica de análisis de contenido. Los resultados obtenidos que demuestran que en el interés y el conocimiento del diagnóstico de ASD por pediatras, todavía no es suficiente para realizar este diagnóstico y para proporcionar una intervención temprana.

Palabras-clave: Autismo. Diagnóstico. Intervención temprana. 


\begin{abstract}
The diagnosis of Autism Spectrum Disorder is performed by clinical evaluation, considering the criteria established by the Diagnostic and Statistical Manual of Mental Disorders (DSM V). The objective of this study was to identify the perceptions of pediatricians about the diagnosis and early intervention in this disorder. Eight pediatricians participated in the study. Data were collected using a sociodemographic questionnaire and a semi-structured interview. To analyze the data, the content analysis technique was used. The results showed that despite advances in the interest and knowledge of the diagnosis of ASD by pediatricians, it was still not enough for them to perform this diagnosis and to provide early intervention.
\end{abstract}

Keywords: Autism spectrum disorder. Diagnosis. Early intervention.

\section{Introdução}

Este estudo versa sobre os sinais de Transtorno do Espectro Autista (TEA), apresentados durante os primeiros dois anos de vida da criança. Geralmente o transtorno é diagnosticado após os três anos de idade, embora já se saiba que há sinais que podem indicar que o bebê está em risco psíquico por apresentar traços autísticos. Os estudos sobre a identificação precoce dos riscos mostram um significativo avanço científico e contribuem para que a intervenção precoce possa iniciar nos primeiros meses de vida do bebê, embora no cenário brasileiro haja carência de instituições especializadas em atendimento interdisciplinar para bebês (Adurens \&Mello, 2017).

O diagnóstico de autismo é essencialmente clínico e leva em consideração os critérios estabelecidos pelo Manual Diagnóstico e Estatístico de Transtornos Mentais (DSM, $5^{\mathrm{a}}$ edição) (American Psychiatric Association [APA], 2014). Assim, a avaliação é realizada a partir da observação da criança e de entrevistas com os pais e cuidadores. A versão atual do DSM-5 centra o diagnóstico em duas premissas: a comunicação social/aspectos qualitativos da reciprocidade socioemocional e o comportamento. Conforme Schmidt (2017) é significativa a heterogeneidade das características apresentadas em cada caso e pode variar desde dificuldades discretas até os casos mais severos, nos quais há um aparente desinteresse pelas pessoas.

Atualmente, estudos têm apontado para o aumento de casos de TEA em todo o mundo. Nos Estados Unidos (EUA) existem hoje um caso para cada 110 pessoas (Brasil, 2014). No que se refere ao Brasil, estima-se que haja 2 milhões de pessoas com esse transtorno (Oliveira, 2011). Sua causa ainda é desconhecida pela ciência, mas, a tendência atual é admitir a existência de fatores de risco genéticos, biológicos e ambientais (APA, 2014). A grande variabilidade dos comportamentos e capacidades cognitivas indica que a etiologia do autismo está relacionada a razões multifatoriais, o que revela destaque para os estudos que envolvem os endofenótipos no autismo (Schmidt, 2017).

Geralmente é a família que primeiramente observa que o desenvolvimento da criança está atípico. Em uma pesquisa realizada no México com 42 crianças com diagnóstico de autismo foi verificado que em $59,5 \%$ dos casos foram os pais que identificaram os primeiros sintomas, em $21,4 \%$, foram os professores e somente em $2,4 \%$ foi o médico pediatra, apesar de ser esse profissional que tem a maior oportunidade de estar em contato com a criança e observar seu desenvolvimento. Quanto às suspeitas dos pais de que algo não estava bem no desenvolvimento de seus filhos, essas aconteceram quando eles tinham por volta de um ano e dez meses, idade mínima de sete 
meses e máxima de cinco anos. Ressalta-seque esses pais passaram por um mínimo de cinco profissionais de saúde até que se estabelecesse o diagnóstico de seus filhos (SampedroTobón, González-González, Vélez-Vieira \&Lemos-Hoyos, 2013).

Além disso, ainda neste mesmo estudo, muitas crianças receberam mais de um diagnóstico até receber o definitivo de TEA. Entre esses diagnósticos, estão o de Transtorno de Déficit de Atenção e Hiperatividade (TDAH), atraso de desenvolvimento, incapacidade intelectual e também de criança "má". Dos diagnósticos de TEA, $35,7 \%$ foram realizados por neuropediatras, seguidos pelos psicólogos (31\%), fonoaudiólogos $(11,9 \%)$, pediatras $(4,8 \%)$ e psiquiatras $(2,4 \%)$ (SampedroTobón, González-González, Vélez-Vieira \& Lemos-Hoyos, 2013).

Isso não é diferente no Brasil. Estudo, no qual foram entrevistados pediatras no Rio Grande do Sul, apontou que a maioria deles desconhecia os sinais de risco que tornaria possível um diagnóstico precoce de autismo (Flores \&Smeha, 2013). Igualmente, Visani e Rabello (2012) mencionaram que os diagnósticos de autismo são realizados tardiamente devido à insegurança e ao desconhecimento dos sinais de risco por parte dos profissionais de saúde que atendem o bebê, principalmente pelos médicos da primeira infância. No estudo desses autores, $78,6 \%$ dos pais detectaram precocemente que algo estava errado com seus filhos, sendo que a principal percepção foi a ausência de linguagem. Apesar disso, o diagnóstico formal apenas aconteceu depois da criança ter passado por até seis ou mais profissionais e/ou instituições de saúde. Sendo que na maioria dos casos $(21,4 \%)$ se passaram três anos entre a referida percepção dos pais e o primeiro diagnóstico. Devido a isso, 28,6\% dos casos de autismo receberam um diagnóstico formal somente aos quatro anos de idade.

Uma das atividades desenvolvidas pelo médico pediatra no Brasil é a puericultura. Essa atividade visa monitorar o crescimento da criança, observando e identificando prováveis problemas que possam interferir no seu desenvolvimento (Zeppone, Volpon \&Del Ciampo, 2012). Alguns pediatras já reconhecem a importância do diagnóstico precoce do TEA, visto que eles são os primeiros profissionais a entrarem em contato com a criança que está apresentando os sinais do transtorno. Apesar disso, também mencionam o desafio que é realizá-lo, pela própria heterogeneidade do transtorno, bem como pela falta de conhecimentos adquiridos na formação profissional. Eles consideram pequena a evolução que as crianças com esse transtorno apresentam nas intervenções profissionais e por isso é um grande desafio acreditar na estimulação precoce (AgripinoRamos \& Salomão, 2014). Ainda que a ideia não seja um consenso, a intervenção precoce singularizada é fundamental para o desenvolvimento da criança com TEA. Sendo assim, é importante que toda suspeita trazida pelos familiares seja investigada pelos médicos, com vistas a um diagnóstico correto (Hernández, Otero \&Rodríguez, 2015).

A importância deste estudo justifica-se pela complexidade do cenário atual, no qual há um número crescente de crianças com autismo (Adurens \&Mello, 2017; Schmidt, 2017) e estudos evidenciando a falta de preparo dos pediatras para o diagnóstico precoce (Agripino-Ramos \& Salomão, 2014; Flores \&Smeha, 2013; Visani \&Rabello, 2012). Soma-se a isso a publicação da Lei Federal $\mathrm{n}^{\circ}$ 13.438, de 26 de abril de 2017 (Brasil, 2017). Ela torna obrigatória a adoção pelo Sistema Único de Saúde (SUS) de protocolo que estabeleça padrões para a avaliação de riscos para o desenvolvimento psíquico. Segundo a nova norma, todos os bebês, nos seus primeiros 18 meses de vida, deverão ser avaliados em consulta pediátrica, por meio de protocolo ou outro instrumento construído com a finalidade de facilitar a detecção de risco para o seu desenvolvimento psíquico.

Considera-se que a implementação dessa determinação modificará a atuação da pediatria no Brasil em relação à saúde psíquica na infância. Por isso, emergem as seguintes 
questões: Como os pediatras estão percebendo a sua atuação diante do Transtorno do Espectro Autista? Por que muitas crianças com autismo não são diagnosticadas pelo pediatra, mas, pelo neuropediatra?

Diante desses questionamentos e da escassez de estudos brasileiros sobre o papel do pediatra no diagnóstico deste transtorno, este estudo buscou identificar as percepções dos médicos pediatras sobre o diagnóstico e a intervenção precoce no Transtorno do Espectro Autista.

\section{Método}

O presente estudo envolve uma pesquisa qualitativa, exploratória e transversal. A população de estudo foi constituída por oito médicos pediatras, provenientes de instituições públicas e privadas. Esses médicos atuam em um município de médio porte, com número de habitantes, estimados para o ano de 2006, de
277.309 pessoas, no estado do Rio Grande do Sul (Instituto Brasileiro de Geografia e Estatística, 2010). A coleta de dados ocorreu no primeiro semestre de 2017. Inicialmente, foram contatados 16 pediatras, mas, somente oito aceitaram participar da pesquisa. Como justificativa para não participarem, os profissionais alegaram não ter tempo disponível para a entrevista ou não possuírem conhecimentos sobre a temática. Para garantir o sigilo quanto à identidade dos participantes, eles se encontram identificados com a letra $\mathrm{P}$ (Pediatra) e o número da ordem cronológica na qual foram realizadas as entrevistas.

Na coleta de dados foram utilizados os seguintes instrumentos: questionário sociodemográfico contendo dados como: idade, sexo, especializações/residências, tempo de formado, e local de trabalho (Tabela $1)$.

Tabela 1 - Caracterização dos Participantes

\begin{tabular}{|c|c|c|c|c|c|}
\hline Profissionais & $\begin{array}{l}\text { Idade } \\
\text { (anos) }\end{array}$ & Sexo & $\begin{array}{l}\text { Especializações/Residências } \\
\text { (em Pediatria e outros) }\end{array}$ & $\begin{array}{l}\text { Tempo de } \\
\text { Formado } \\
\text { (anos) }\end{array}$ & $\begin{array}{l}\text { Local de } \\
\text { trabalho }\end{array}$ \\
\hline P1 & 55 & $\mathrm{~F}$ & $\begin{array}{l}\text { Residência em Pediatria e } \\
\text { Especialização em Saúde } \\
\text { Coletiva }\end{array}$ & 32 & $\begin{array}{l}\text { Pronto } \\
\text { Atendimento e } \\
\text { Hospital }\end{array}$ \\
\hline $\mathrm{P} 2$ & 65 & $\mathrm{~F}$ & $\begin{array}{c}\text { Residência em Pediatria e } \\
\text { Especialização em Saúde } \\
\text { Pública }\end{array}$ & 43 & $\begin{array}{l}\text { Consultório } \\
\text { Particular e } \\
\text { Aposentadoria } \\
\text { da Rede } \\
\text { Pública de } \\
\text { Saúde }\end{array}$ \\
\hline P3 & 68 & M & Residência em Pediatria & 34 & $\begin{array}{c}\text { Hospital, } \\
\text { Consultório } \\
\text { Particular e } \\
\text { Pronto } \\
\text { Atendimento }\end{array}$ \\
\hline $\mathrm{P} 4$ & 56 & M & $\begin{array}{l}\text { Residência em Pediatria e } \\
\text { especialização em } \\
\text { Emergências Pediátricas }\end{array}$ & 32 & $\begin{array}{l}\text { Hospital e } \\
\text { Consultório } \\
\text { Particular }\end{array}$ \\
\hline P5 & 64 & $\mathrm{~F}$ & Residência, Mestrado e & 34 & Coordenação \\
\hline
\end{tabular}


Doutorado em Pediatria

\begin{tabular}{|c|c|c|c|c|c|}
\hline P6 & 53 & $\mathrm{~F}$ & $\begin{array}{c}\text { Residência e Doutorado em } \\
\text { Pediatria }\end{array}$ & 28 & $\begin{array}{l}\text { Hospital e } \\
\text { Consultório } \\
\text { Particular }\end{array}$ \\
\hline P7 & 34 & $\mathrm{~F}$ & $\begin{array}{l}\text { Residência em Pediatria e } \\
\text { Especialização em } \\
\text { Infectologia Pediátrica }\end{array}$ & 09 & $\begin{array}{l}\text { Hospital e } \\
\text { Clínica } \\
\text { Particular }\end{array}$ \\
\hline P8 & 48 & $\mathrm{~F}$ & $\begin{array}{l}\text { Residência em Pediatria e } \\
\text { Especialização em Saúde } \\
\text { Coletiva }\end{array}$ & 19 & $\begin{array}{l}\text { Unidade } \\
\text { Básica de } \\
\text { Saúde e Pronto } \\
\text { Atendimento }\end{array}$ \\
\hline
\end{tabular}

de Curso

Universitário

Hospital e

Consultório

Hospital e

Clínica seguintes categorias: conhecimento sobre os

Igualmente, foi utilizada a entrevista semiestruturada a partir das seguintes questões norteadoras: Você poderia me dizer quais são os sinais de risco para o autismo que um bebê pode apresentar? Com que idade é possível fazer esse diagnóstico? Como o pediatra pode dar a notícia do diagnóstico aos pais? Você acha que é importante uma intervenção precoce nesse transtorno? Você recebeu conhecimentos sobre sinais de risco e critérios para o diagnóstico de TEA na sua formação? Você já atendeu algum bebê que apresentava sinais de risco para o autismo? Foi você ou os pais que perceberam os primeiros sintomas? Já fez algum diagnóstico de TEA? Que instrumentos utilizou e quanto tempo levou para concluí-lo? Como você se sentiu na situação de informar aos pais o diagnóstico? Se isso já foi há algum tempo, você faria de forma diferente hoje? Se você fizesse o diagnóstico de TEA hoje, quais os encaminhamentos que faria, considerando o contexto e recursos locais? Você tem conhecidos, amigos ou familiares que tenham filhos com esse diagnóstico?

Para analisar os dados foi utilizada a técnica de análise de conteúdo proposta por Bardin (2011). Dessa forma, em uma primeira etapa foi realizada uma leitura flutuante, que consistiu na leitura inicial das falas, buscando a identificação dos principais significados que os participantes do estudo atribuíram a cada tema em todas as entrevistas, listados como categorias. Ao final da análise emergiram as sinais de risco; atuação do pediatra na realização do diagnóstico; percepção dos pediatras sobre a intervenção precoce e profissionais envolvidos.

O estudo adotou todas as questões éticas de pesquisa envolvendo seres humanos. Seu projeto foi submetido ao Comitê de Ética do Centro Universitário Franciscano e aprovado, segundo registro CEP/UNIFRA: 1.876.868/2016, parecer $\mathrm{n}^{\circ} 2196779$. Todos os participantes assinaram o Termo de Consentimento Livre e Esclarecido (TCLE).

\section{Resultados e discussões}

Sete dos oito profissionais entrevistados apontaram como principais sinais de risco para o autismo que um bebê pode apresentar, a falta de interação com a mãe, com o próprio pediatra e com o ambiente em geral, manifestada pela ausência de contato visual e a não resposta aos estímulos. Já na criança mais velha, a não aquisição ou o retardo da linguagem, as estereotipias e movimentos repetitivos também despertam preocupação nesses profissionais. A pediatra (P2) mencionou o distúrbio do sono, a irritabilidade, a hipotonia e a hiperatividade, como possíveis sinais indicativos do transtorno, conforme ilustra a sua fala: " $U m$ dos principais sinais é que não te olha, a hiper... o sono, distúrbio do sono... aí tem crianças que... a irritabilidade, ou o contrário, ou muito hiperativo ou muito, muito paradões, 
sabe? Muito molinho". Conforme P3: "Quando a criança tem quatro, cinco meses, às vezes demora um pouquinho mais, ela não me olha, eu olho para ela, coloco uma lanterna, e ela está ali...essa daí, não é que é risco, é possibilidade de ter autismo". A P5 salientou dificuldades na relação com a mãe [...] no início a falta de interação mãe-bebê, principalmente, no olhar. Apenas uma das entrevistadas (P8) alegou não ter nenhum conhecimento dos sinais de risco: "Sinais de risco para o autismo? Não sei”.

Percebeu-se que os pediatras entrevistados, principalmente a $\mathrm{P}(2)$, já apresentavam conhecimentos, ainda que precários, dos sinais de risco para o autismo, se comparado com um estudo anteriormente desenvolvido nesse mesmo contexto. Nesse estudo, os médicos evidenciaram desconhecer sinais, tais como, distonias musculares, recusa do olhar, perturbação do sono, intolerância alimentares e outros (Flores \&Smeha, 2009). Segundo Vasconcelos (2009) o não olhar do bebê para a mãe é um dos sinais mais indicativos de risco de autismo, pois é a partir do olhar e da fala da mãe que surge a subjetividade da criança. $\mathrm{Na}$ presente pesquisa o "não olhar" foi o sinal de risco mais citado pelos profissionais, o que pode indicar um progresso, ainda que sutil, no interesse desses profissionais por esse transtorno. Cabe ressaltar que a pediatra (P2) que apresentou mais conhecimento dos sinais de risco é a única que tem familiar próximo com esse transtorno, o que pode explicar essa diferença.

É importante que o pediatra esteja preparado para identificar os sinais de risco do autismo, para fazer o diagnóstico formal e conhecer as intervenções terapêuticas disponíveis para o encaminhamento dos pais, pois assim é possível a realização de uma intervenção com vistas a otimizar 0 desenvolvimento desses bebês. Vasconcelos (2009) referiu que, se o pediatra ao detectar o não olhar do bebê para a mãe o encaminhasse para consultas psicológicas especializadas, resultados satisfatórios no desenvolvimento desses bebês seriam obtidos. Para isso, seria relevante o trabalho em conjunto entre os psicólogos e os profissionais da primeira infância. Mas, infelizmente, a realidade percebida não é essa. Os diagnósticos são desenvolvidos tardiamente, pelo fato dos pediatras nem sempre considerarem aspectos psíquicos na avaliação do bebê e, também, dada a escassez de profissionais da psicologia nos serviços públicos e privados.

Os profissionais entrevistados evidenciaram acreditar que devem observar o desenvolvimento da criança desde os primeiros meses, buscando perceber se o desenvolvimento não está de acordo com o esperado para determinada idade. Porém, na criança com mais idade é mais fácil perceber e ter certeza de que os comportamentos não são resultados da influência do ambiente no qual ela vive.

Dois anos a gente já começa a ter uma suspeita mais consistente, mas, o grande problema da criança pequena é a gente conseguir filtrar aquilo que é interferência do meio, inferência do meio, um meio que às vezes não estimula adequadamente, um meio que protege demais a criança. $(\mathrm{P} 4)$

[...] Então ele não sorri na idade que tem que sorrir, que já seria mais o social, mais assim, não senta na idade adequada. Então, é mais ou menos nos marcos do desenvolvimento neuropsicomotor que agente se dá conta que está atrasado e que daí tu pode pensar em autismo. [...] Não é assim uma idade... mais assim...quanto mais velha a criança, mais fácil de fazer o diagnóstico. (P5)

Sobre isso, autores sugerem que analisar vídeos familiares pode auxiliar na identificação de sinais de risco mais sutis no primeiro ano de vida do bebê, como aqueles de ordem afetiva. A interação mãe-bebê é caracterizada pela expressão do afeto e o período de transição da interação diática para a atenção compartilhada, geralmente, ocorre entre 9-12 meses de idade (Garcia \&Lampreia, 
2011). Igualmente, a observação do pais, principalmente no que tange à interação social do bebê, é importante para que aconteça o diagnóstico precoce. Acredita-se que a atenção compartilhada se desenvolve na interação com as outras pessoas e culmine no desenvolvimento da fala. Portanto, mesmo que os pais não tenham observado isso, o pediatra deve estar atento a esse comportamento do bebê, para que seja possível uma intervenção precoce (Zanon, Backes \&Bosa, 2014).

A psicanálise, na tentativa de auxiliar na detecção dos referidos sinais, desenvolveu o instrumento contendo Indicadores Clínicos de Risco para o Desenvolvimento Infantil (IRDI). Esse instrumento é constituído por 31 indicadores, relacionados com os quatro eixos de formação do sujeito psíquico, e pode ser aplicado por pediatras em consultas de rotina. O IRDI tem capacidade de avaliar se a constituição psíquica da criança está no seu curso normal ou apresenta riscos. Destaca-se a importância do uso do referido instrumento, uma vez que se os problemas forem detectados precocemente é possível reverter o quadro proporcionando à criança um desenvolvimento normal, impedindo, assim, a instalação de um transtorno psíquico (Kupfer et al., 2009).

\section{Atuação do Pediatra na Realização do Diagnóstico}

A grande maioria dos pediatras entrevistados relatou já ter atendido crianças que apresentavam sinais de risco para o autismo. Na maioria das vezes foram eles que perceberam os primeiros sintomas. Sendo assim, raramente os sinais foram percebidos pelos pais. Segundo alguns dos participantes, a família costuma negar a situação, trocando, frequentemente, de pediatra em busca de uma notícia melhor. P1 referiu: "Já tive um bebê que eu suspeitei, vários questionamentos para a família, a família negando essa questão".

[...] mas, existe muita resistência dos pais, por isso que às vezes eles passam de pediatra em pediatra. Quer dizer, os pais resistem e aí tu acaba encaminhando para o especialista, para o especialista conseguir convencer a família. (P7)

Sim. Os pais negaram por muito tempo. [...] Eu tenho... acho que um que nunca mais voltou depois que eu falei nisso. Era meu paciente regular. [...] E aí eu falei para eles para investigar e eles pararam de vir aqui e foram para outra clínica e eu não soube mais. (P6)

Apesar do relato desses profissionais, já é considerado como aspecto relevante para o referido diagnóstico precoce do TEA valorizar os relatos dos pais, todas as vezes que estes trazem preocupações quanto a comportamentos atípicos dos filhos (Mesquita \&Pegoraro, 2013). Segundo Zanon, Backes \&Bosa (2014) os comportamentos atípicos mais relatados coincidem com as três áreas de comprometimento do TEA. Os autores mencionaram que em primeiro lugar é relatado atraso ou alguma peculiaridade referente à linguagem. Em segundo lugar, problemas na interação social, seguidos por comportamentos estereotipados e repetitivos. Também relataram dificuldades na alimentação, sono, bem como no desenvolvimento motor. Evidenciando, dessa forma, a importância do profissional que está acompanhando a criança, considerar e investigar as preocupações dos pais. É importante que o pediatra pergunte aos pais detalhes sutis do comportamento do seu filho, pois o fato dos pais não expressarem preocupação, não significa que eles não tenham nenhuma (Sudhinaraset \&Kuo, 2013).

Em outros país estambém se acredita na importância de valorizar os relatos. Artigo mexicano traz que os pais são os primeiros a observar os sinais de risco para o autismo. Assim, 50\% os observam nos primeiros 12 meses de vida. Porém, há atraso na busca dos profissionais para realizar o diagnóstico precoce. Dessa forma, menos de 38\% das famílias receberam diagnóstico pelos serviços de saúde. Os autores consideraram importante a criação de treinamentos para os profissionais da educação e da saúde para a identificação 
dos sinais de risco precocemente (MárquezCaraveo \&Albores-Gallo, 2011).

Ainda sobre essa questão, entre o pediatra e os pais há uma relação de bastante proximidade. Os pais confiam e dependem dele para aliviar suas preocupações e angústias referentes ao desenvolvimento de seu filho. Sendo assim, esse profissional pode utilizar essa relação para buscar a contribuição dos pais para o processo de diagnóstico precoce do TEA (Sudhinaraset \&Kuo, 2013). Por outro lado, há de se considerar que a confiança depositada no pediatra por parte dos pais, pode ser interrompida pelo fato de perceberem a insegurança desse profissional na realização do diagnóstico. Isso pode ter sido a causa do descrédito dos pais, relatado pelos pediatras (P1, P6 e P7) e o abandono do atendimento relatado pela pediatra $\mathrm{P} 6$.

Também, os participantes do presente estudo concordaram de forma unânime que não compete a eles efetuar o diagnóstico do autismo. Alegaram que devem acompanhar o desenvolvimento da criança desde o seu nascimento, observando sinais de risco para esse transtorno. Uma vez que percebam que esse desenvolvimento está atípico, devem encaminhar para um profissional especialista na área. Sendo assim, o papel desse profissional seria de suspeitar do TEA e encaminhar para o neuropediatra realizar o diagnóstico. Minha conduta é sempre a mesma. Encaminhar para o neuro fechar um diagnóstico. Eu não digo para os pais que "o seu filho tem autismo". Nunca tive essa conduta assim (P1). Alguns que eu fiz ... na verdade, a gente faz a suspeita diagnóstica e a gente acaba encaminhando para o neuropediatra para ele fazer a confirmação (P4). Eu encaminho para o neuro. Suspeito, digo os meus motivos, mas, quem tem que fazer esse diagnóstico é o neuro, ele segue alguns critérios que o pediatra geral não está habituado a usar (P6). [...] eu não faço diagnóstico porque acho que é necessário um especialista dar esse diagnóstico. Já que é um diagnóstico mais difícil. Eu acredito que esse é o papel do especialista (P7).
A dificuldade e insegurança dos pediatras para realizar o diagnóstico do autismo podem estar relacionadas a ser recente a preocupação com a saúde mental das crianças. Ela foi introduzida no contexto brasileiro, principalmente, pela Reforma Psiquiátrica e o Estatuto da Criança e do Adolescente, que ampliaram os direitos nessa fase da vida. Ressalta-se a importância dessa mudança, pois é na infância que se torna possível identificar grande parte dos transtornos e se intervir para um melhor desenvolvimento desses indivíduos. Porém, para que essas mudanças se concretizem é imprescindível, também, uma modificação no currículo de formação em medicina e residência em pediatria, para que os profissionais inseridos no mercado de trabalho estejam preparados para atuar no diagnóstico do TEA e outros transtornos observáveis na infância (Menezes \&Melo, 2010). Na presente pesquisa, ainda que não se possa afirmar com certeza, pois somente uma das entrevistadas (P7) se formou recentemente, parece que as universidades estão buscando atingir esse patamar.

No que concerne ao encaminhamento dos bebês com suspeita de autismo para o neuropediatra fazer o diagnóstico, Sudhinaraset \&Kuo (2011) apontaram que o atraso no diagnóstico se deve, entre outros fatores, principalmente, as referências pediátricas para atendimento especializado. $\mathrm{O}$ excesso de serviços frequentados pelos pais em busca de esclarecimento dos sinais identificados contribuem para esse atraso e oneram os sistemas de saúde. Uma vez que o diagnóstico seja realizado pelos pediatras se reduzirá o tempo entre ele e a intervenção precoce, podendo obter melhores resultados no desenvolvimento da criança e redução nos custos dos serviços de saúde.

Apesar disso, ainda que não seja no contexto brasileiro, autores trazem que é importante inserir aspectos psicossociais nas consultas pediátricas, pois esses aspectos são poucos valorizados no primeiro ano de vida. Porém, para eles o diagnóstico do autismo é multiprofissional, sendo importante a 
participação de psicólogos, pediatras, fonoaudiólogo, neuropediatras, psiquiatras e pedagogos. Para que esses profissionais atuem, deve ser facilitado o acesso das famílias a eles. Também, faz-se imprescindível que um protocolo seja criado para não haver repetição de ações, dificultando e atrasando mais o diagnóstico precoce. Também, é importante uma formação adequada de todas as categorias com vistas ao diagnóstico e intervenção precoce nesse transtorno (Sevilla, Bermúdez \&Sánchez, 2013).

Seguindo nessa mesma linha, no Brasil também se acredita que além da pediatria, é importante que outras categorias se envolvam no diagnóstico precoce do autismo, principalmente, a psicologia. Entretanto, em uma revisão de literatura desenvolvida no país, verificou-se que apenas 34\% dos autores de 19 artigos tinham formação nessa área e apenas três desses artigos foram publicados em revistas de psicologia. Isso pode indicar, assim como no caso dos pediatras, a falta de interesse pela temática por parte dos psicólogos ou o não preparo desses profissionais para atuarem na área. Sendo assim, é imprescindível que esse tema seja abordado na graduação de psicologia, considerando as características de cunho psicológico desse transtorno e a possibilidade da psicologia trabalhar em conjunto com os pediatras na realização do referido diagnóstico (Mesquita \&Pegoraro, 2013).

Ao perceber que o desenvolvimento da criança está atípico os pediatras precisam dar essa notícia aos pais. Assim, eles procuram fazer isso paulatinamente para não causar maiores impactos. Os entrevistados informaram acreditar que um diagnóstico de autismo é algo muito angustiante para a família, uma vez que não há prognóstico de cura. Sobre o diagnóstico $\mathrm{P} 2$ relatou: Tem que ir com cautela. Alguma coisa está acontecendo, o bebê está diferente, né? Então tem que fazer uma investigação, né? Pedir um auxílio do neuropediatra e outros profissionais da saúde, né? Mas não... eu acho que é muito importante não assustar. Para $\mathrm{P} 4$ a informação aos pais não deve ocorrer de forma abrupta: A gente tem que ir trabalhando aos poucos, a gente tem que ir mostrando gradualmente as diferenças de comportamento, a gente tem que expor as dificuldades da criança, de forma que os pais não consigam negar. Conforme $\mathrm{P} 3$, os sintomas e o diagnóstico não devem ser elucidados em um único momento: Tem que dar em parcelas. Eu sempre digo que pode ser, não é? Pois eles já até consultaram alguém, alguém já sugeriu. Até um parente, ou a professora já... É mais ou menos por aí.

Informar o diagnóstico para os pais é angustiante também para os pediatras entrevistados. Eles relataram não se sentir bem, porque preferem sempre dar boas notícias. Apesar disso, acreditam que melhoraram na sua forma de dar a notícia com o passar do tempo e atribuem isso à experiência profissional e a novos conhecimentos, adquiridos em cursos sobre a temática. P7 referiu que: Eu me senti angustiada também. Porque é uma doença que não tem cura, porque é bastante sofrido esse processo de adaptar essa criança na sociedade. Então, eu fiquei desconfortável também. P2, P5 e P6 exemplificaram, por meio de suas falas, a experiência diante do diagnóstico de TEA em um paciente:

Você sabe que dá uma certa tristeza no pediatra. É uma coisa assim que a gente vive com a família, o pediatra é o médico da família. Então, realmente a gente fica abalado. $\dot{E}$ como se fosse filho da gente. (P5)

Eu acho que estou mais sensivel que no passado, eu era mais fria, parece. Não sei se quando a gente é mais nova...era mais impetuosa, você vai adquirindo mais experiência, você vai mudando, tu pede auxílio para outro profissional estar junto para dizer, principalmente da psicologia. (P2) 
Melhorou. Olha vou te dizer que teve muito a ver com um curso que a gente fez aqui o ano passado. [...] Então, a partir dali eu me senti mais segura, assim de suspeitar e falar da minha suspeita, mesmo quando não tenho razão, mesmo estando errada. (P6)

Referente a essa questão, estudo apontou que realmente os médicos vivenciam muita tristeza na hora de dar a notícia aos pais da deficiência de uma criança. Não somente a tristeza, mas, angústia, solidariedade e frustração, também são sentimentos igualmente relatados por esses profissionais. Este último sentimento pode estar relacionado ao fato de não ser possível, no caso das deficiências intelectuais, a cura com um tratamento medicamentoso, como na maioria das patologias que eles diagnosticam. Há de se considerar, também, que esses profissionais na sua formação não são preparados para dar esse tipo de notícia (Luisada, Fiamenghi Junior, Carvalho, Assis-Madeira, \&Blascovi-Assis, 2015).

O não preparo desses profissionais para dar essa notícia, limita-os na hora de passar as informações para os familiares dessas crianças. As informações repassadas são pessimistas e limitantes, ampliando a angústia e a ansiedade desses pais. É importante que esses profissionais sejam preparados para passar as devidas informações aos pais, de uma forma que apresentem a eles as possibilidades de desenvolvimento do seu filho. Assim, esses pais terão esses profissionais como suporte para ajudá-los na superação das dificuldades (Lemes, \&Barbosa, 2007). Pode-se perceber a importância do preparo do profissional na fala da pediatra (P6), que depois de ter passado por uma capacitação se sentiu mais segura para comunicar suas suspeitas aos pais.

Quase todos os pediatras entrevistados relataram não ter recebido, durante sua formação profissional,conhecimentos sobre os sinais de risco e critérios para realizar o diagnóstico do autismo, exceto a pediatra (P7), formada mais recentemente. P3 mencionou:
Não, nunca. Mesmo quando eu fiz residência. P6 corroborou: Na minha formação, não. $\mathrm{Na}$ minha formação de faculdade, mas, de cursos e congressos que a gente participa.

Não. Na verdade há trinta e poucos anos atrás, quando eu fiz a minha graduação, essa área comportamental era... não só o Transtorno do Espectro Autista, as outras coisas também que tinha a ver com psiquiatria, com psicologia infantil, eram poucos explorados. (P4)

Tenho bastante leitura, artigos, tenho um ou dois livros sobre autismo em casa, porque é um assunto prevalente na clínica. Eu que faço pediatria geral se eu não souber manusear minimamente as coisas do autismo eu vou frustrar muito meus pacientes. (P4)

Sim. Nós tivemos na pediatria, sim. Os diagnósticos foram aos poucos, foram mudando, começaram a ver que existe...não era tão fixo, que existem vários graus de autismo, mas, a gente... se fala muito disso, se estuda muito autismo. (P7)

É importante que seja fortalecida a formação dos profissionais de saúde da primeira infância, principalmente, com conteúdos de psicologia e transtornos do desenvolvimento. Os protocolos de crescimento e desenvolvimento infantil deveriam ser modificados, incluindo neles a valorização do desenvolvimento psíquico tanto quanto o desenvolvimento físico dos bebês (Sampedro-Tobón, González-González, Vélez-Vieira \&Lemos-Hoyos, 2013).

Um programa de treinamento desenvolvido nos EUA mostrou que é possível preparar esses médicos para realizar o diagnóstico precoce do autismo. Esse programa treinou vários profissionais de saúde que atuavam na pediatria em um contexto pediátrico comunitário, dentre eles vinte 
pediatras. Na avaliação pós treinamento foi possível perceber mudanças significativas na atuação desses profissionais. Assim, houve um aumento de $85 \%$ na identificação desse transtorno em sua prática clínica (Swanson et al., 2013).

No Brasil, houve aumento na oferta de informação sobre esses transtornos e seu diagnóstico, pois a maioria dos pediatras participantes da presente pesquisa relataram ter participado em congressos, cursos ou terem lido artigos sobre essa temática. No entanto, apesar da pediatra (P7) relatar que recebeu conhecimentos sobre o diagnóstico do autismo em sua formação, não há diferença na postura em relação à realização do diagnóstico e à intervenção precoce, quando comparada a de outros médicos pediatras entrevistados. Isso pode indicar que, mesmo diante de novos conhecimentos na formação acadêmica, ainda o interesse dos pediatras é voltado para as doenças que são passíveis de cura, principalmente, a cura por meio do tratamento medicamentoso.

Quando questionados sobre quais instrumentos são utilizados para fazer o diagnóstico do autismo a maioria dos profissionais entrevistados concordou que o diagnóstico deve ser feito pela observação, história e exame clínicos. Somente dois pediatras (P2 e $\mathrm{P} 4)$ relataram ter conhecimento de algum tipo de questionário utilizado na realização do diagnóstico: Não me lembro quantas perguntas, mas, é um protocolo que tem as questões sobre o autismo (P2). $\mathrm{P} 4$ disse possuir o instrumento, mas, não utiliza por não ter sido capacitado para isso: Eu até tenho no computador, mas, eu não uso tanto, até porque, como eu te disse assim, isso tem que ter um treino, uma regularidade de uso de instrumentos maior do que eu costumo ter. $\mathrm{P} 4$ foi o único profissional que citou o DSM $5^{\mathrm{a}}$ edição, como manual que traz os critérios para a realização do diagnóstico, mas, ele informou não concordar com tais critérios, pois, para ele, essa ferramenta acaba incluindo como autistas pessoas que não possuem o transtorno.
[...] Então, essa é a minha... ainda a minha divergência com alguns métodos diagnósticos, alguns critérios diagnósticos que são para mim muito abrangentes $e$ vagos. [...] A ferramenta que nós médicos, pediatras, principalmente usamos, as ferramentas do DSM V, são inclusivas demais. Tem algumas personalidades aí que não são exatamente autistas e que são rotuladas como tal, podem ser rotuladas como tal. Ta? (P4)

O Manual de Diagnóstico e Estatística de Transtornos Mentais (DSM) é o recurso mais importante para fins de diagnósticos psiquiátricos, seja pelo psiquiatra ou o clínico geral. De acordo com o DSM-5, déficits em apenas os dois primeiros domínios essenciais são necessários para o diagnóstico, a saber: déficits na comunicação social e na interação social. Assim, o atraso no desenvolvimento da linguagem não é mais necessário para o diagnóstico. No entanto, o principal obstáculo à aplicação imediata desses critérios na prática clinica, provavelmente, será a disponibilidade de ferramentas de diagnóstico de acordo com os novos critérios. Os critérios utilizados para o diagnóstico do autismo ainda se encontram aquém de serem interpretados como definitivos, mas há de se considerar a importante contribuição desse manual para o reconhecimento do referido transtorno (Artigas-Pallares \&Paula, 2012).

Além desse manual, muitos instrumentos são utilizados para identificar e caracterizar o autismo, um deles é o Autism Behavior Checklist (ABC), que consiste em uma listagem de comportamentos não adaptativos na qual são identificados e somados os valores para se traçar um perfil comportamental e também uma análise da severidade do TEA. Quanto mais altos os valores, maior a probabilidade desse transtorno. Outro instrumento bastante importante é a Childhood Autism Rating Scale (CARS). Trata-se de uma escala que contribui na identificação e diferenciação dos diversos graus de TEA em portadores do transtorno. 
Em uma análise desses dois testes, aplicados simultaneamente em um grupo de crianças e adolescentes com diagnóstico de TEA, verificou-se que o CARS pode falhar na questão de não diagnosticar algumas crianças autistas, mas, o ABC pode incluir como autistas crianças com outros transtornos. Desse modo, os instrumentos podem falhar quanto à concordância, mas, quanto mais severo for o quadro do TEA, mais há convergência entre eles. Sendo assim, é importante que os dois instrumentos sejam utilizados de forma complementar como auxiliares no diagnóstico do transtorno citado (Santos et al., 2012).

Além desses instrumentos, utiliza-se também, principalmente para rastreamento e triagem do TEA, o Modified Checklist for Autism in Toddlers (M-Chat). O mencionado instrumento foi adaptado e validado no Brasil. É composto por 23 questões que podem ser aplicadas por qualquer profissional de saúde. As referidas questões exigem respostas "sim" ou "não" e são aplicadas para os pais de bebês de 18 a 24 meses de idade, com o objetivo de averiguar a presença de comportamentos conhecidos como sinais de risco para o TEA (Brasil, 2014).

$\mathrm{Na}$ presente pesquisa, o fato dos pediatras não terem comentado sobre esses instrumentos parece indicar falta de conhecimento ou insegurança no manuseio deles como mencionou o profissional P4. Assim, no cenário atual é imprescindível que os pediatras busquem aprofundar seus conhecimentos sobre o transtorno.

\section{A Percepção dos Pediatras sobre a Intervenção Precoce}

Todos os pediatras entrevistados relataram que é importante uma intervenção precoce no TEA e esta deve ser realizada por uma equipe multidisciplinar, para que se obtenham melhores resultados no desenvolvimento das crianças portadoras desse transtorno. Eles também citaram várias categorias profissionais que poderiam estar envolvidas nessa intervenção. Os profissionais mencionados não foram somente os da saúde, mas, também os da educação e, inclusive, pessoas que trabalham para as famílias dessas crianças.

É muito importante essa intervenção precoce onde vai se trabalhar com fonoaudióloga, terapeuta ocupacional, fisioterapeuta, neurologista, pediatra, psicólogo...quer dizer, a equipe realmente para tratar o autismo, é uma equipe multiprofissional e...quanto mais cedo for o diagnóstico, maior a chance que a criança vai ter de conviver satisfatoriamente com a sociedade. (P1)

Sim. A intervenção precoce que é multidisciplinar, que não é só médico. A intervenção do terapeuta, $a$ intervenção da escola, $d a$ psicopedagoga, é muito importante e quanto mais cedo, melhor a resposta. (P6)

Todos. Tudo que está junto aí, pediatra é o primeiro que deve perceber, eu acho que depois tem que ir no neuropediatra fazer uma avaliação neurológica, psicólogo, professor, pais, até pessoas da casa, até uma empregada pode ajudar a acompanhar, eu acho que é multiprofissional, né? (P3)

Apesar disso, dois desses pediatras (P3 e P5), embora também acreditassem na importância da intervenção precoce, relataram que não perceberam bons resultados dessa intervenção. Segundo um deles, as crianças evoluem muito pouco, ainda que assistidas por longo tempo pela equipe de profissionais interventores. A pediatra (P5) lamentou não ter um medicamento para tratar o transtorno.

Eu acho que sim. Quando mais precoce, melhor. Só que eu não sei que grau de evolução tem. Eu não tenho encontrado muitas... muita melhora. [...] Não é uma evolução... é 
uma criança que vai ficar com dificuldades, vai ter...vai ser difícil aprender, deve aprender menos, vai ter dificuldades de muita ordem. [...] não tenho visto assim grandes progressos, tem uma melhora boa, mas, não aquela evolução assim... vai... vai desaparecer essa... essa, essa síndrome, sempre vai ficar com retardo. $(\mathrm{P} 3)$

Com certeza. Difícil é o tratamento, mas, ... é difícil o tratamento, porque não tem uma medicação ainda, bom se a gente tivesse uma pilulazinha para isso, mas, não existe. Então assim, é multiprofissional... do fisio...Depende, assim, do que tu nota que está mais atrasado, né? (P5)

De acordo com a literatura, a intervenção precoce melhora a qualidade de vida das crianças com TEA, principalmente, quando estes chegam na idade adulta. Essa intervenção é mais eficaz quando é realizada por uma equipe multidisciplinar. $\mathrm{O}$ tratamento pode ser organizado conforme a idade ou peculiaridade da criança, tendo como focos principais, a interação social, a atenuação de comportamentos desadaptativos, assim como o desenvolvimento da linguagem precocemente (Fonseca \&Missel, 2014; Mesquita \&Pegoraro, 2013). Na sua maioria, os benefícios alcançados se referem ao campo da linguagem. Assim, 20\% a 30\% dos autistas desenvolvem a fala quando são estimulados precocemente (Mesquita \&Pegoraro, 2013).

Considerando que os custos do tratamento dos transtornos psíquicos são elevados, pois requer a assistência de uma equipe multidisciplinar especializada, que deve ser constante e prestada por toda a vida, é imprescindível que um diagnóstico precoce seja realizado com o intuito de que o retorno e o avanço no desenvolvimento dessas crianças seja maior e em um menor espaço de tempo. Esse mesmo autor considerou que uma vez que o diagnóstico do autismo seja precoce, é possível desenvolver um plano terapêutico que pode resultar na redução dos comprometimentos no processo de desenvolvimento da criança, ainda que essa criança sofra intervenção somente a partir dos três anos de idade, os ganhos no seu desenvolvimento são bastante perceptíveis e positivos. Quanto mais cedo ocorra a intervenção, maior possibilidade de otimizar os resultados e reduzir os referidos custos (Moraes, 2011).

No que concerne à intervenção da psicologia, principalmente da abordagem psicanalítica, Bernardino e Kupfer (2008) apontaram que o elemento organizador do desenvolvimento típico de uma criança é o surgimento do sujeito psíquico. Para que a criança se constitua como tal é necessário que ela seja inscrita pela família no campo da linguagem e da cultura. Isso ocorre gradualmente a partir da função materna organizada em torno de quatro eixos: o primeiro se relaciona com a suposição do sujeito. Nele a mãe antecipa o sujeito psíquico, que ainda não se constituiu no bebê, principalmente, pela manifestação de palavras denominadas mamanhês. Essa forma da mãe falar causa prazer no bebê fazendo com que ele busque corresponder a essa antecipação na tentativa de trazer esse prazer de volta. O segundo eixo se refere às primeiras reações involuntárias do bebê, como por exemplo, o choro. Uma vez que a mãe reconhece essa demanda e a satisfaz, estabelece-se uma demanda de amor que a criança poderá usar na base de sua linguagem e nos relacionamentos futuros com qualquer pessoa.

Quanto ao terceiro eixo, ele é caracterizado pela alternância presença/ausência da mãe. As ações da mãe, relacionadas com o referido eixo, visam tornar o bebê um sujeito desejante, ou seja, um ser autônomo e singular. Para isso, ele terá que experimentar a descontinuidade entre a sua demanda e a satisfação proporcionada por quem desempenha a função materna. É nesse espaço de tempo que ele pode se experimentar como sujeito. O quarto eixo, por sua vez, se associa com a função paterna de fazer a castração, causando a separação mãe-bebê e dessa forma impedindo que a pessoa que 
desempenha a função materna considere o bebê como seu "objeto" de satisfação. Isso fará com que a criança se diferencie da mãe e se torne um sujeito singular. Dessa forma, quando se instalam, por qualquer motivo, falhas no surgimento do sujeito psíquico, são causados problemas que interferirão no desenvolvimento da criança.

Sendo assim, para essa abordagem, o diagnóstico precoce do autismo não visa rotular a criança, mas sim, abrir a possibilidade de intervenção em uma estrutura que ainda pode ser modificada. Conclusões de estudos mostraram que os resultados são melhores se a intervenção acontece antes dos três anos de vida, idade na qual é preconizado o diagnóstico definitivo. A intervenção no campo do outro com a criança, melhora a interação do bebê com a mãe. Geralmente, as crianças com diagnóstico de autismo somente são encaminhadas ao psicanalista quando já se encontram na faixa etária escolar, precisamente depois de ter se concluído o diagnóstico médico de uma doença. Dessa forma, muitos sintomas poderão já se encontrar cristalizados, dificultando ou até mesmo, inviabilizando a intervenção. Essa área da psicologia necessita intervir nos sinais iniciais para, principalmente, evitar que a patologia se instale (Vasconcelos, 2009). Considerando os discursos dos pediatras deste estudo, o psicólogo foi citado como um profissional a ser considerado na intervenção precoce. Apesar disso, não se pode afirmar que eles acreditem nessa possibilidade, pois a inclusão desse profissional pode ter ocorrido pela pesquisadora para representar a área de conhecimento.

\section{Considerações Finais}

O estudo relatado neste artigo buscou identificar as percepções dos médicos pediatras sobre o diagnóstico e intervenção precoce no Transtorno do Espectro Autista (TEA). A maioria dos profissionais entrevistados mostrou conhecimento, ainda que superficial, dos sinais de risco para o autismo. Também, relataram serem os primeiros a perceberem os sinais de risco para o TEA nos seus pacientes, mas, afirmaram que não conseguem a colaboração dos pais no entendimento do problema. Além disso, os pediatras deste estudo afirmaram sentir-se inseguros na hora de realizar o diagnóstico do TEA, por isso delegam essa atribuição a especialistas como o neuropediatra. Eles justificaram pelo fato de não terem recebido conhecimentos sobre esse transtorno na formação acadêmica e nem na residência em pediatria. No entanto, considerando que a maioria dos profissionais fez seus cursos de graduação há mais de uma década, pode-se salientar que, na época, pouco se sabia sobre o autismo, realidade diferente da atual, na qual o TEA vem sendo muito estudado e há publicações científicas recentes e muito relevantes.

Em consideração ao exposto, destacase a necessidade de atualização constante dos profissionais da área médica, pois os conhecimentos adquiridos durante suas formações não são suficientes para as necessidades da clinica pediátrica contemporânea. Quanto ao diagnóstico do TEA, o conhecimento raso poderá ampliar a dificuldade de conversar com os pais sobre o desenvolvimento atípico de seus filhos. Somase a isso o desconforto referido pelos profissionais deste estudo, diante da necessidade de tratar uma criança que poderá ter limitações severas no seu desenvolvimento. O prognóstico manifestado pela maioria dos pediatras não é otimista. Consideraram que a intervenção precoce pode minimizar os sintomas do transtorno, mas, sem apresentar grandes progressos. Isso pode explicar o insipiente anseio do pediatra quanto à detecção dos sinais precoces nos primeiros anos de vida da criança.Apesar dos avanços no interesse dos pediatras pelo autismo, constata-se que o conhecimento adquirido ainda não alicerça o fechamento do diagnóstico.Ao encaminhar o paciente para um neuropediatra, muitas vezes, a família encontra dificuldades de conseguir uma consulta na rede pública com este especialista. Isso pode postergar muito o conhecimento dos pais sobre o diagnóstico e, 
em decorrência, perde-se tempo para iniciar uma estimulação precoce.

É importante que essa temática seja abordada atualmente nos cursos de formação em medicina e residência pediátrica para que sejam formados profissionais com capacidade de reconhecer os sinais precoces de risco psíquico, realizar o diagnóstico e acompanhar a saúde de uma criança com o transtorno, pois há especificidades, como, por exemplo,no comportamento e na alimentação, que precisam ser compreendidas pelo pediatra. Em geral, os pais possuem uma importante relação de confiança com o pediatra de seus filhos, por isso, buscam solucionar dúvidas por meio de questionamentos a esse profissional. Ele é referência para a família no que se refere a melhor forma de cuidar e promover $\mathrm{o}$ desenvolvimento de sua criança. A responsabilidade do pediatra está em informar corretamente a família sobre o transtorno e sobre as possíveis formas de intervenção, mantendo uma relação empática e estimulando os pais a serem proativos diante do tratamento e das necessidades da criança em cada fase do seu desenvolvimento.

\section{Referências}

Adurens, F.D.L. \&Mello, M.S. (2017).

Reflexões acerca da possibilidade de prevenção do autismo. Estilos clin., São Paulo, 22(1), p. 150-165.

Agripino-Ramos, C. S, \&Salomão, N. M. R. (2014). Autismo e síndrome de Down: concepções de profissionais de diferentes áreas. Psicol. Estud, 19 (1), p. 103-114.

American Psychiatric Association (APA). (2014). Manual de Diagnóstico e Estatística de Trantornos DSM-V. Porto Alegre: ARTMED.

Artigas-Pallares, J.,\& Paula I. (2012). El autismo 70 anos después de Leo Kanner y Hans Asperger. Rev. Asoc. Esp. Neuropsiq. 32 (115), p. 567-587.

Bernardino L. M. F., \&Kupfer, M. C. M. (2008). A criança como mestre do gozo da família atual: desdobramentos da "pesquisa de indicadores clínicos de risco para o desenvolvimento infantil. Ver. Mal-Estar e Subj. VIII (3), p. 661-680.

Bardin, L. (2011). Análise de Conteúdo. São Paulo: Edições 70.

Brasil. Ministério da Saúde. Secretaria de Atenção à Saúde. (2014). Departamento de Ações Programáticas Estratégicas.

Diretrizes de Atenção à Reabilitação da Pessoa com Transtornos do Espectro do Autismo (TEA). Brasília: Ministério da Saúde.

Brasil. Estatuto da criança e do adolescente: Lei federal $\mathrm{n}^{\circ} 13.438$, de 26 de abril de 2017, DF: Senado Federal.

Flores, M. R.,\&Smeha, L. N. (2013). Bebês com risco de Autismo: o não olhar do médico. Ágora. XVI (n. especial), p. 141157.

Fonseca, S. A., \&Missel, A. (2014). Autismo: auxilio ao desenvolvimento antecipadamente. Revista Pós-Graduação: Desafios Contemporâneos. Faculdade Cesuca.1 (1), p. 83-99. Recuperado de http://ojs.cesuca.edu.br/index.php/revposgr aduacao/article/view/622.

Garcia M. L., \&Lampreia, C. (2011). Limites e possibilidades da identificação de risco de Autismo no primeiro ano de vida. Psicol.: Reflex. e Crít., 24 (1), p. 300-308.

Hernàndez, O. C., Otero, D. L., \&Rodríguez, N. C. (2015). Autismo: uma cercamiento hacia el diagnóstico y la genética. Rev. Ciências. Médicas, 19 (1), p. 157-178.

Instituto Brasileiro de Geografia e Estatística. Censo Demográfico. (2010). Cidades: IBGE. Recuperado de www.IBGE.gov.br/home/cidades.

Kupfer, M. C. M., Jerusalinsky A. N., Bernardino L. M. F., Wanderley D., Rocha, P. S. B., Molina, S. E., Sales, L. M., Stellin, R., Pesaro, M. E., Lerner, R. (2009). Valor preditivo de indicadores clínicos de risco para o desenvolvimento infantil: um estudo a partir da teoria psicanalítica. Lat. Am. Journal of Fund. Psychopath. Online.6 (1) 48-68. Recuperado de http://www.fundamentalpsychopathology.o rg/uploads/files/latin_american/v6_n1/valor preditivo_de indicadores_clinicos_de ris co_para_o_desenvolvimento_infantil.pdf. 
Lemes L. C., \& Barbosa M. A. M. (2007). Comunicando a mãe o nascimento do filho com deficiência. Acta Paul Enferm. 20(4), p. 441-445.

Luisada V, Fiamenghi-Jr G.A, Carvalho S.G., Assis-Madeira E.A., \&Blascovi-Assis S.M. (2015) Experiências de médicos ao comunicarem o diagnóstico da deficiência de bebês aos pais. Ver Ciên Saúde. 8 (3), p. 121-128.

Márquez-Caraveo, \& M. Albores-Gallo, L. (2011). Austistics pectrum disorders: diagnostic and therapeutic challenges in Mexico. Salud Mental. (34), p. 435-441.

Menezes, T. T., \& Melo, V. J. (2010). O pediatra e a percepção dos transtornos mentais na infância e adolescência. Adoles Saude.7 (3), p. 38-46.

Mesquita, W. S., \&Pegoraro, R. F. (2013). Diagnóstico e tratamento do transtorno autístico em publicações brasileiras: revisão de literatura. J. Health Sci Inst. 31 (3), p. 324-329.

Moraes, C. de. Autismo Infantil: aspectos clínicos e epidemiológicos. (2011). Rev. Debat. Psiquiatr. Associação Brasileira de Psiquiatria 1 (2), p. 6-8.

Oliveira, C. (2011). Um retrato do autismo no Brasil. Rev. Espaço Aberto. Ed 170. São Paulo: USP.

Sampedro-Tobón, M. E., González-González, M., Vélez-Vieira, S., \& Lemos-Hoyos, M. (2013). Detección temprana em transtornos del espectro autista: uma decisión responsable para um mejor pronóstico. Bol. Med. Hosp. Infant. Mex.70 (6), p. 456-466. Santos, T. H. F., Barbosa, M. R. P., Pimentel, A. G. L., Lacerda, C. A., Balestro, J. I., Amato, C. A. de la H., Fernandes, F. D. M. (2012). Comparação dos instrumentos Childhood Autism Rating Scale e Autism Behavior Checklist na identificação e caracterização de indivíduos com distúrbios do espectro autístico. J. Soc. Bras. Fonoaudiol. 24 (1), p. 104-106.

Schmidt, C. (2017). Transtorno do Espectro Autista: onde estamos e para onde vamos? Psicologia em Estudo. 22(2), p. 221-230.

Sevilla, M. del S. F., Bermúdez, M. O. E. \&Sánchez, J. J. C. (2013). Detección temprana del Autismo: profesionales implicados. Rev. Esp. Salud Publica. 87 (2), p. 191-199.

Sudhinaraset, A. \&Kuo, A. (2013). Parent's perspectives on the role of Pediatricians in Autism Diagnosis. J Autism Dev Disord. 43, p. 747-748.

Swanson, A., Warren, Z. E., Stone, W. L., Vehorn, A. C. , Dohrmann, E. \&Humberd, Q. (2013). The diagnosis of autism in community pediatric settings: Does advanced training facilitate practice change?.Autism. 18 (5), p. 555-561.

Vasconcelos, R. M. de A. R. L de. (2009). Autismo Infantil: a importância do tratamento precoce. XV Encontro Nacional da ABRAPSO/76. Psicologia e Políticas de Existência: Fronteiras e Conflitos. Anais. Maceió: ABRAPSO.

Visani, P., \& Rabello, S. (2012). Considerações sobre o diagnóstico precoce na clínica do autismo e das psicoses infantis. Rev. Latinoam. Psicopat. Fund., 15 (2), p. 293-308.

Zanon, R. B., Backes, B., \&Bosa, C. A. (2014). Identificação dos primeiros sintomas do Autismo pelos pais. Psicol. Teor. e pesq. 30 (1), p. 25-33.

Zeppone, S. C., Volpon, L. C., \&Del Ciampo, L. A. (2012). Monitoramento do desenvolvimento infantil realizado no Brasil. Rev Paul Pediatr. 30 (4), p. 594599.

Dados sobre as autoras:

- Maria Evanir Vicente Ferreira é Enfermeira - Mestrado em Saúde Coletiva Universidade Estadual de Londrina, UEL. Graduanda no curso de Psicologia do Centro Universitário Franciscano-UNIFRA.

- Luciane Najar Smeha é Psicóloga - Docente no curso de graduação em Psicologia e no Mestrado em Saúde Materno Infantil do Centro Universitário Franciscano - UNIFRA. Mestrado e Doutorado em Psicologia pela PUC-RS. 